\title{
Implementation of an advance directive focus in a Chronic Multi- Organ Rare Disease Clinic
}

\author{
Nancy Elorreaga ${ }^{1}$, Deanna Allred ${ }^{1,2}$, Gloria Ortiz ${ }^{1}$, Constance McNeill ${ }^{1}$, Mary Beth Scholand ${ }^{3}$, Tracy \\ M. Frech ${ }^{2,4}$
}

${ }^{1}$ University of Utah Hospital and Clinics, Salt Lake City, UT, USA; ${ }^{2}$ Division of Rheumatology, ${ }^{3}$ Division of Pulmonary and Critical Care, Department of Internal Medicine, University of Utah, Salt Lake City, UT, USA; ${ }^{4}$ Division of Rheumatology, Department of Internal Medicine, Salt Lake Veterans Affair Medical Center, Salt Lake City, UT, USA

Contributions: (I) Conception and design: N Elorreaga, D Allred, MB Scholand, TM Frech; (II) Administrative support: D Allred, G Ortiz, C McNeill, MB Scholand, TM Frech; (III) Provision of study materials or patients: D Allred, G Ortiz, C McNeill, MB Scholand, TM Frech; (IV) Collection and assembly of data: N Elorreaga, D Allred, MB Scholand, TM Frech; (V) Data analysis and interpretation: N Elorreaga, TM Frech; (VI) Manuscript writing: All authors; (VII) Final approval of manuscript: All authors.

Correspondence to: Tracy M. Frech, MD, MS. Division of Rheumatology, Department of Internal Medicine, University of Utah, 4b200 SOM, 30N 1900 E, Salt Lake City, UT 84132, USA. Email: tracy.frech@hsc.utah.edu.

\begin{abstract}
Background: Education about an advance directive is frequently not addressed in the outpatient, ambulatory care setting. The objective of this study was to identify patients that do not have an advance directive in a Chronic Multi-Organ Rare Disease Clinic model, and define the role of a social worker in providing advance care planning (ACP).

Methods: The Chronic Multi-Organ Rare Disease Clinic incorporates a multi-disciplinary team to provide outpatient care to over 600 patients. A review of advance directives filed in the electronic health record (EHR) prior to hiring a clinic social worker was examined in this high risk population.

Results: A total of 15 patients (2\%) of this patient population were identified as having a completed, active advance directive filed with their EHR prior to hiring a clinic social worker. The clinic social worker began ACP discussions and inquiries about the status of patient advance directives with a total of 162 patients during September 2016-April 2017. Of these 162 patients, 14 patients (8.6\%) submitted their completed advance directives for filing with their EHR after advanced care discussions were initiated by the clinic social worker. Two patients who completed an advance directive, died during this 7 -month time period. Only three patients declined to complete advance directives during this same time period.

Conclusions: Patient-centered care must incorporate ACP. A clinic social worker is an effective member of a multi-disciplinary team and can incorporate education about advance directives in order to improve health care quality.
\end{abstract}

Keywords: Advance directives; rare disease; patient-centered care

Submitted Jul 31, 2017. Accepted for publication Aug 03, 2017.

doi: 10.21037/apm.2017.08.06

View this article at: http://dx.doi.org/10.21037/apm.2017.08.06

\section{Introduction}

A goal for a Chronic Multi-Organ Rare Disease Clinic is to provide health care focused on patient-centered goals, reduction in the number of inappropriate tests and treatments, and assessment of meaningful outcomes with an effective multi-disciplinary team (1). Health care reform efforts have identified advance directives as a critical aspect of quality (2). Clinical discussions should include a focus on whether life prolongation is a goal, circumstances under which it would no longer be a goal, and the trade-offs each 
person would be willing to make between length and quality of life; however, time constraints may limit the ability of this discussion to take place. Integration of a social worker, who is knowledgeable about advanced directives, into a Chronic Multi-Organ Rare Disease Clinic can improve health risk assessment and patient-centered longitudinal health planning.

Advanced directives allow a patient's right to selfdetermination of what actions will be taken upon his or her body in the health care context, including health care proxy authority for decision making in the event of incapacity (3). Overall, advanced directives provide health care providers, patients, and patient families with control over care desires at the end of life. There are two general categories for advance directives, instructional directive and proxy directive, and three general types of advance directives, a health care agent/power of attorney/proxy, a living will/health care directive, and provider orders, which can include provider order for life-sustaining treatment (POLST) and do not resuscitate (DNR) order. Instructional directives, like living wills, DNRs, and POLSTs, clarify the patients' end-of-life care wishes. A living will documents an individual's preferences for end-of-life care-the medical care that a person does and/or does not want under certain medical conditions and is used when an individual is unable to communicate these wishes. The designation of a proxy decision maker identifies the surrogate a patient has chosen to communicate his/her end-of-life care wishes in situations when the patient is unable to communicate. It is common for pre-made advance directive forms to include both the instructional directives and the designated proxy decision maker; however, a health care provider must understand these documents in order to discuss them (3).

Patient-centered strategies for chronic disease require tighter adherence to evidence-based clinical practice guidelines and more effective teamwork (4). Unfortunately, the majority of people who will receive end-of-life care will have this care provided in the acute hospital setting and from physicians who do not know them (5). Most people do not initiate conversations with their family/ loved ones or physicians about their end-of-life care goals, values, and preferences, which is possibly due to patients waiting for healthcare professionals to initiate advanced care planning discussions (6). One study identified that the comprehensiveness of the provider-lead advanced care planning discussion is a contributing factor for patientcompleted advance directives (7). Thus, healthcare professionals need to take on a more active role with their patients' advance care planning (ACP), including: end-oflife care decisions, ongoing ACP, ensuring patients have a completed, up-to-date advance directive, and discussing the importance of patients having in-depth discussions about their end-of-life care wishes with their appointed surrogates.

\section{Methods}

The University of Utah Health Chronic Multi-Organ Rare Disease Clinic incorporated social work-initiated ACP discussions during initial and follow-up outpatient clinic appointments. Semi-structured interviews, based on the informed-consent process, were conducted during these visits starting in September 2016, when the social worker joined the healthcare team. In addition, the clinic social worker conducted follow-up contact through subsequent clinic visits, the electronic health record (EHR) messaging system, or letter correspondence approximately every 2 months following the initial contact and ACP discussion. Inquiries regarding patients' knowledge of advance directives, status of patients' completed advance directives, and inclusion of patients' completed advance directives with their EHRs as well as the importance of having in-depth discussions about patients' end-of-life care wishes with appointed surrogates and/or ongoing ACP were included in the initial and subsequent follow-up contacts. In addition, reminders regarding the importance of reviewing completed advance directives yearly and with a health or significant life change were given on initial and subsequent clinic visits to any patient who had a completed advance directive filed in their EHR. An initial report was generated to identify the Chronic Multi-Organ Rare Disease Clinic patients who had an advance directive filed with their EHR as well as ongoing monthly reports to identify any additional patients who have subsequently filed their advance directive with their EHR. A basic tracking spreadsheet was developed to assist the clinic social worker with identifying which patients had initial and follow-up contact, had completed advance directives, had filed advance directives with their EHR, had declined to complete/submit an advance directive, and were due for follow-up contacts.

\section{Results}

The Chronic Multi-Organ Rare Disease Clinic incorporates a multi-disciplinary team to provide outpatient care to over 600 patients. A total of 15 patients ( $2 \%$ of the clinic's 
patient population) were identified as having a completed, active advance directive filed with their EHR prior to having any ACP discussions with the clinic social worker. For a 7 -month period, between 09/14/16-04/14/17, the clinic social worker began ACP discussions and inquiries about the status of patient advance directives with a total of 162 patients. Of these 162 patients, 14 patients or $8.6 \%$ submitted their completed advance directives for filing with their EHR after ACP discussions were initiated by the clinic social worker. Two patients, who identified that they did not have a completed advance directive, died during this 7-month time period. In addition, three patients declined to complete advance directives during this same time period and following ACP discussions.

\section{Discussion}

An outpatient clinic social worker is well-positioned to initiate and continue advanced care planning discussions with patients due to their extensive training in interpersonal skills, their experience and ability to approach and discuss difficult topics, and their access to training for advanced care planning and end-of-life care issues. In addition, the utilization of a social worker rather than physiciandirected advanced care planning discussions appears to be less threatening to patients since anecdotal experience has indicated that patients often wonder if physicians are withholding information regarding their prognosis when a physician addresses the status of patients' advance directives. Furthermore, physician time-constraints and knowledge of the ACP may provide a limitation for this discussion. Although the effects of culture, race, and ethnicity need to be further explored and reviews of patient-completed and submitted advance directives as well as ongoing ACP discussions need to be further studied, our preliminary assessment supports that a comprehensive, on-going ACP program in a high risk outpatient clinic by a clinic social worker is effective. The goal of our Chronic Multi-Organ Rare Disease Clinic is to demonstrate an improvement in quality of patient-centered care through increasing the rate of advance directives filed in patients' EHRs.

\section{Acknowledgements}

Funding: Frech K23: research reported in this publication was supported by the National Institute of Arthritis and Musculoskeletal and Skin Diseases of the National Institutes of Health under Award Number K23AR067889. The content is solely the responsibility of the authors and does not necessarily represent the official views of the National Institutes of Health. VA Merit: the project described was supported by Award Number I01CX001183 from the Clinical Science Research \& Development Service of the VA Office of Research and Development.

\section{Footnote}

Conflicts of Interest: The authors have no conflicts of interest to declare.

Ethical Statement: The study was approved by institutional ethics board of the University of Utah (IRB: 38705). Written informed consent was obtained from the patient for publication.

\section{References}

1. Allred DA, Frech TM, McComber C, et al. Chronic Multi-organ Rare Disease: The role of the nurse practitioner as a leader of the health care team. J Med Pract Manage 2017.

2. Mold J. Goal-Directed Health Care: Redefining Health and Health Care in the Era of Value-Based Care. Cureus 2017;9:e1043.

3. Basanta WE. Advance directives and life-sustaining treatment: a legal primer. Hematol Oncol Clin North Am 2002;16:1381-96.

4. Mitka M. Patient-centered medical homes offer a model for better, cheaper health care. JAMA 2012;307:770-1.

5. Plonk WM Jr, Arnold RM. Terminal care: the last weeks of life. J Palliat Med 2005;8:1042-54.

6. Blackford J, Street AF. Facilitating advance care planning in community palliative care: conversation starters across the client journey. Int J Palliat Nurs 2013;19:132-9.

7. Aoki T, Miyashita J, Yamamoto Y, et al. Patient experience of primary care and advance care planning: a multicentre cross-sectional study in Japan. Fam Pract 2017;34:206-12.

Cite this article as: Elorreaga N, Allred D, Ortiz G, McNeill C, Scholand MB, Frech TM. Implementation of an advance directive focus in a Chronic Multi-Organ Rare Disease Clinic. Ann Palliat Med 2017;6(Suppl 2):S206-S208. doi: 10.21037/ apm.2017.08.06 